\title{
ON THE RATE OF GROWTH OF THE WALSH ANTIDIFFERENTIATION OPERATOR
}

\author{
R. PENNEY
}

\begin{abstract}
In [1] Butzer and Wagner introduced a concept of differentiation and antidifferentiation of Walsh-Fourier series. Antidifferentiation is accomplished by convolving (in the sense of the Walsh group) against a function $\Omega$. In this paper we study growth and the continuity properties of $\Omega$ showing that $\Omega$ is bounded from below by -1 , is continuous in $(0,1)$ and grows at most like $\log 1 / x$ as $x \rightarrow 0$. We use this information to study continuity properties of differentiable functions.
\end{abstract}

Introduction. In [1], Butzer and Wagner introduced a concept of derivative and antiderivative of Walsh-Fourier series. In this paper we are interested primarily in the antiderivative.

Let $W_{n}$ denote the $n$th Walsh function. Let $\Omega$ be the a.e. defined function whose Walsh-Fourier series is

$$
\Omega(x)=1+\sum_{K=1}^{\infty} \frac{W_{K}(x)}{K}
$$

( $\Omega$ exists as an $L^{2}$ function since $1 / K$ as in $l^{2}$ ). Then convolution against $\Omega$ with respect to the Walsh addition on $[0,1]$ defines an integral operator which in the Butzer-Wagner theory plays the role of antidifferentiation.

It is the purpose of this paper to investigate the continuity properties and growth properties of $\Omega$. Our main results are that $\Omega$ is continuous everywhere in $[0,1)$ except at 0 and at zero it grows at most like $\log 1 / x$. Furthermore, we show that $\Omega(x) \geqq-1$ for all $x$. This is interesting for, as commented in [2], $\Omega$ is not positive. Hence, the antiderivative of a positive function need not be positive. However, from the above convolution with $1+\Omega$ is positive and still yields a concept of antiderivative. Hence, it is possible to get a positive antidifferentiation operator.

Our main technique is to compare $\Omega$ with the function

$$
\tilde{\Omega}(x)=\sum_{K=0}^{\infty} \frac{W_{K}}{K+1}(x) .
$$

The relationship between $\tilde{\Omega}$ and $\Omega$ is simple:

$$
|\Omega(x)-\tilde{\Omega}(x)|=\left|\sum_{K=1}^{\infty}\left(\frac{1}{K}-\frac{1}{K+1}\right) W_{K}(x)\right| \leqq \sum_{K=1}^{\infty} \frac{1}{K}-\frac{1}{K+1}=1 .
$$

Received by the editors December 9, 1974.

AMS (MOS) subject classifications (1970). Primary 42A56.

Key words and phrases. Walsh functions, antidifferentiation. 
(The series telescopes!) Furthermore, since the above sum converges absolutely, $\Omega-\tilde{\Omega}$ is continuous in the Walsh sense so $\Omega$ will be continuous whenever $\tilde{\Omega}$ is.

The advantage to studying $\tilde{\Omega}$ over $\Omega$ is that we can write down a formula for $\tilde{\Omega}$. Specifically, we reason as follows. Let $t \in \mathbf{R},|t|<1$. Let $P(t, x)$ $=\sum_{K=0}^{\infty} t^{K} W_{K}(x)$. This converges absolutely and uniformly in $|t| \leqq \delta$ $<1$ for all $x$.

In this paper we derive the following formula:

$$
P(t, x)=\frac{1}{1-t} \prod_{K=0}^{\infty}\left(\frac{1-t^{2_{K}}}{1+t^{2_{K}}}\right)
$$

where $x \in(0,1)$ and $x=\sum_{K=0}^{\infty} 2^{-i_{K}-1}$ is the diadic expansion of $x$ (if $x$ is a diadic rational we take the finite expansion). From this formula, $\lim _{t \rightarrow 1^{-}} P(t, x)$ exists. Hence, $\lim _{t \rightarrow 1^{-}} \int_{0}^{t} P(s, x) d s$ exists. It follows that $\sum W_{K}(x) /(K+1)$ is Abel summable. Furthermore, $K W_{K}(x) /(K+1) \geqq-1$ for all $K$. It follows from the Hardy-Littlewood theorem [3, Theorem 4.22] that $\sum W_{K}(x) /(K+1)$ converges for all $x \in(0,1)$, and equals $\int_{0}^{1} P(t, x)$. This allows us to obtain our explicit estimates on $\tilde{\Omega}$.

We begin with the proof of (1) above. We consider the $W_{n}$ as having been extended periodically to all of $\mathbf{R}$. Let

$$
P_{N}(t, x)=\sum_{K=0}^{2 N-1} t^{K} W_{K}(x)
$$

LEMMA 1 . If $N \geqq i \geqq 0$,

$$
P_{N}\left(t, x+2^{-(i+1)}\right)=P_{i}(t, x) P_{N-i}\left(-t^{2^{i}}, 2^{i} x\right) .
$$

Proof.

$$
\begin{aligned}
P_{N}\left(t, x+2^{-(i+1)}\right) & =\sum_{K=0}^{2^{N}-1} t^{K} W_{K}\left(2^{-(i+1)}\right) W_{K}(x) \\
& =\sum_{l=0}^{2^{N-i}-1} \sum_{m=0}^{2^{i}-1}\left\{W_{m+2^{i} l}\left(2^{-(i+1)}\right) W_{m+2^{i} l}(x) t^{m+2^{i} l}\right\} .
\end{aligned}
$$

Now, observe that $W_{m+2^{i} l}\left(2^{-(i+1)}\right)=(-1)^{l}$ and that $m+2^{i} l=m+2^{i} l$. Hence, the above is

$$
=\sum_{l=0}^{2^{N-i}-1}\left(-t^{2^{i}}\right)^{l} W_{2^{i} l}(x) \sum_{m=0}^{2^{i}-1} W_{m}(x) t^{m} .
$$

The proof is completed by noting that $W_{2^{i} l}(x)=W_{l}\left(2^{i} x\right)$. Q.E.D.

Lemma 2. Suppose $x=\sum_{K=0}^{n} 2^{-i_{K}}$ and $N \geqq i_{n}>i_{n-1}>i_{0}>0$. Then

$$
P_{N}(t, x)=\frac{1-t^{2}}{1-t} \prod_{K=0}^{n} \frac{1-t^{2^{i_{K}-1}}}{1+t^{2^{i_{K}-1}}}
$$

Proof. Note that if $x$ is an integer, $W_{K}(x)=1$ for all $K$ and, hence, $P_{N}(t, x)$ 
is a geometric series which sums to $\left(1-t^{2^{N}}\right) /(1-t)$. Hence, by Lemma 1 ,

$$
\begin{aligned}
P_{N}\left(t, 2^{-i_{0}}\right) & =P_{i_{0}-1}(t, 0) P_{N-i_{0}+1}\left(-t^{2^{i_{0}-1}}, 2^{i_{0}-1} \cdot 0\right) \\
& =\frac{1-t^{2_{0}-1}}{1-t} \frac{1-\left(-t^{2^{i_{0}-1}}\right)^{2^{N-i_{0}+1}}}{1+t^{i_{0}-1}}=\frac{1-t^{2^{N}}}{1-t} \frac{1-t^{2^{i_{0}-1}}}{1+t^{2^{i_{0}-1}}} .
\end{aligned}
$$

(Note that $2^{N-i_{0}+1}$ is even.) Hence, the formula is true if $n=0$. Now, assume it true for all integers less than $n$. Let $x_{0}=x-2^{-i_{n}}$. Then

$$
P_{N}(t, x)=P_{N}\left(t, x_{0}+2^{-i_{n}}\right)=P_{i_{n}-1}\left(t, x_{0}\right) P_{N-i_{n}+1}\left(-t^{i_{n}-1}, 2^{i_{n}-1} x_{0}\right) .
$$

Since $2^{i_{n}-1} x_{0}$ is an integer, the $P_{N-i_{n}+1}$ term equals $\left(1-t^{2^{N}}\right) /\left(1+t^{2_{n}-1}\right)$. Applying the induction hypothesis to the other term and simplifying yields the result. Q.E.D.

We can now prove formula (1) above. If $x$ is a diadic rational, (1) follows from Lemma 2 by letting $N \rightarrow \infty$, so we may suppose that we are using an infinite expansion. Let $x=\sum_{K=0}^{\infty} 2^{-i_{K}}$ where $0<i_{0}<i_{1}<\cdots<i_{j}<\cdots$, and for each $n \in N$ let $x_{n}=\sum_{K=0}^{n} 2^{-i_{K}}$. From the above,

$$
P\left(t, x_{n}\right)=\frac{1}{1-t} \prod_{K=0}^{n}\left(\frac{1-t^{2^{i_{k}-1}}}{1+t^{2^{i_{K}-1}}}\right) .
$$

Since the series for $P$ converges uniformly in $x$ if $|t|<1, P$ is continuous (in the Walsh sense) in $x$ and, hence, $P\left(t, x_{n}\right) \rightarrow P(t, x)$. This proves convergence of the infinite product and formula (1). Q.E.D.

Corollary 1 . $\tilde{\Omega}(x) \geqq 0$ for all $x$ and, hence, $\Omega(x) \geqq-1$ for all $x$.

Proof. As shown in the introduction, $\tilde{\Omega}(x)=\int_{0}^{1} P(t, x) d x$, which is clearly positive. Q.E.D.

COROLlaRY 2. There are constants $C_{1}$ and $C_{2}$ such that $|\Omega(x)| \leqq C_{1} \log 1 / x$ $+C_{2}$ for all $x \in(0,1)$.

Proof. If $a>0,(1-a) /(1+a)<1$. Hence,

$$
P(t, x) \leqq \frac{1}{1-t} \frac{1-t^{2_{0}-1}}{1+t^{2_{0}-1}} \leqq \frac{1}{1-t}\left(1-t^{2^{i_{0}-1}}\right)=\sum_{K=0}^{2^{i_{0}-1}-1} t^{K} .
$$

Hence,

$$
\tilde{\Omega}(x)=\int_{0}^{1} P(t, x) d t \leqq \sum_{K=1}^{2_{0}-1} \frac{1}{K} \leqq C_{1} \log 2^{i_{0}-1}+C_{2} .
$$

But $x \leqq 22^{-i_{0}}=2^{-\left(i_{0}-1\right)}$. Hence, $\log 2^{i_{0}-1} \leqq \log 1 / x$, proving the claim for $\tilde{\Omega}$ and, hence, for $\Omega$. Q.E.D.

REMARKS. Corollary 2 could also be proven analogously to the technique used by Yano [4] to obtain estimates on $\sum W_{K} /(K)^{\alpha}(0<\alpha<1)$. However, lower bounds do not seem to be so easily obtainable from Yano's technique.

Corollary $3 . \Omega$ is continuous on $(0,1)$, and is unbounded as $x \rightarrow 0^{+}$. 
Proof. Note that if $x=\sum_{K=0}^{\infty} 2^{-i_{K}}$ as before, then

$$
P(t, x) \leqq \frac{1}{1-t} \frac{1-t^{2^{i_{0}-1}}}{1+t^{2^{i_{0}-1}}} \leqq \sum_{K=0}^{2^{i_{0}-1}} t^{K} \leqq 2^{i_{0}-1} \quad \text { for } t \in(0,1) .
$$

Also note that

$$
\int_{0}^{1} P(t, x)(1-t) d t=\sum\left(\frac{1}{n+1}-\frac{1}{n+2}\right) W_{n}(x)
$$

is an absolutely convergent Fourier series and, hence, is Walsh continuous.

Now write $x_{n}=\sum_{K=0}^{n} 2^{-i_{K}}$. It follows trivially from (1) that $P(t, x)$ $=P\left(t, x-x_{n}\right)(1-t) P\left(t, x_{n}\right)$. Hence, if $y$ is such that $y_{n}=x_{n}$, then

$$
\begin{aligned}
& \left|\int_{0}^{1} P(t, x)-P(t, y) d t\right| \\
& \quad=\left|\int_{0}^{1}\left\{P\left(t, x-x_{m}\right)(1-t)-P\left(t, y-y_{n}\right)(1-t)\right\} P\left(t, x_{n}\right) d t\right| \\
& \quad \leqq 2^{i_{0}-1}\left\{\int_{0}^{1} P\left(t, x-x_{n}\right)(1-t) d t+\int_{0}^{1} P\left(t, y-y_{n}\right)(1-t) d t\right\} .
\end{aligned}
$$

As $n \rightarrow \infty$ this tends to zero from the continuity of $\int_{0}^{1} P(t, x)(1-t) d t$.

The unboundedness is trivial since

$$
\tilde{\Omega}\left(2^{-(i+1)}\right)=\int_{0}^{1} \frac{1-t^{2^{i}}}{\left(1+t^{2^{i}}\right)(1-t)} d t
$$

which $\rightarrow \infty$ as $i \rightarrow \infty$. Q.E.D.

REMARKS. Ladhawala has constructed a more direct proof of the continuity of $\Omega$ following the proof that $\Omega$ is in $L^{1}$ given in [1]. Note, incidentally, that $\Omega \in L^{1}$ follows trivially from $\Omega \in L^{2}$.

Now, one of the basic properties of differentiation is that if $f$ $\in L^{1}(\mathbf{R})$ and $\lim _{t \rightarrow 0}(f(\cdot+t)-f(\cdot)) / t$ exists in $L^{1}$, then $f$ is absolutely continuous. (One simply integrates this limit to obtain $f$.) The corresponding theorem for Walsh differentiation is false. However, the following is true. The first part was pointed out to us by Ladhawala.

Corollary 4. If $f \in L^{1}([0,1])$ and Df exists in the $L^{1}$ sense (see [1]) and is in $L^{P}$ for some $P>1$, then $f$ is Walsh continuous. However there exist functions in $L^{1}([0,1])$, differentiable in the $L^{1}$ sense, which are not continuous in the Walsh sense.

Proof. By results of [1], $f=\Omega * D f+\hat{f}(0)$ (* in the Walsh sense). Since $\log 1 / x$ is in $L^{q}$ for all $1 \leqq q<\infty$ and $L^{P}$ convolved with $L^{q}$ is continuous $(P$ and $q$ conjugate exponents), the first claim follows

To prove the second part, note that since $\Omega$ is in $L^{2}, \Omega * L^{1} \subset L^{2} \subset L^{1}$. From results of [1], every element of $\Omega * L^{1}$ is differentiable in the $L^{1}$ sense, with derivative in $L^{1}$. Hence, if our theorem is false, convolution by $\Omega$ maps $L^{1}$ into the space of Walsh-continuous functions on $[0,1]$. By the closed graph 
theorem this mapping would have to be continuous from $L^{1}$ into the uniform topology on $C([0,1])$. In particular, $f \rightarrow \Omega * f(0)=\int_{0}^{1} f(x) \Omega(x) d x$ is continuous in $L^{1}$, implying that $\Omega$ is essentially bounded, which is false by Corollary 3. Q.E.D.

ACKNOWLEDGEMENTS. The author would like to acknowledge the help of Ladhawala for his pointing out the work of Butzer and Wagner to us and for his frequent and lengthy discussion of this work with us.

\section{REFERENCES}

1. Butzer and Wagner, Walsh series and the concept of a derivative, Applicable Anal. 3 (1973), $29-46$.

2. - On a Gibbs-type derivative in Walsh-Fourier analysis with applications, Technical Report of the Technological University of Aachen, Aachen, West Germany.

3. Powell and Shah, Summability theory and applications, Van Nostrand Reinhold, London, 1972.

4. S. Yano, On Walsh-Fourier series, Tôhoko Math. J. (2) 3 (1951), 223-242. MR 13, 550.

Department of Mathematics, Purdue University, West Lafayette, Indiana 47907 